OR relating diabetes to LTBI. Similar results were obtained when the analysis was restricted to contacts.

Conclusions In the current PREDICT cohort, diabetes does not appear to be associated with LTBI after adjustment for age. The relationship between diabetes and TB disease observed elsewhere may reflect an increased risk of disease rather than infection.

\section{S58 TB CO-INFECTION IS ASSOCIATED WITH POOR SURVIVAL AMONG HIV INFECTED PATIENTS IN ENGLAND AND WALES}

${ }^{1} \mathrm{D}$ Zenner, ${ }^{1} \mathrm{~S}$ Conti, ${ }^{1} \mathrm{Z}$ Yin, ${ }^{1} \mathrm{M}$ Kall, ${ }^{2} \mathrm{M}$ Kruijshaar, ${ }^{1} \mathrm{~B}$ Rice, ${ }^{1} \mathrm{M}$ Lipman, ${ }^{3} \mathrm{~A}$ Pozniak, ${ }^{1} \mathrm{I}$ Abubakar, ${ }^{1} \mathrm{~V}$ Delpech; ${ }^{1}$ Centre for Infectious Disease Surveillance and Control Public

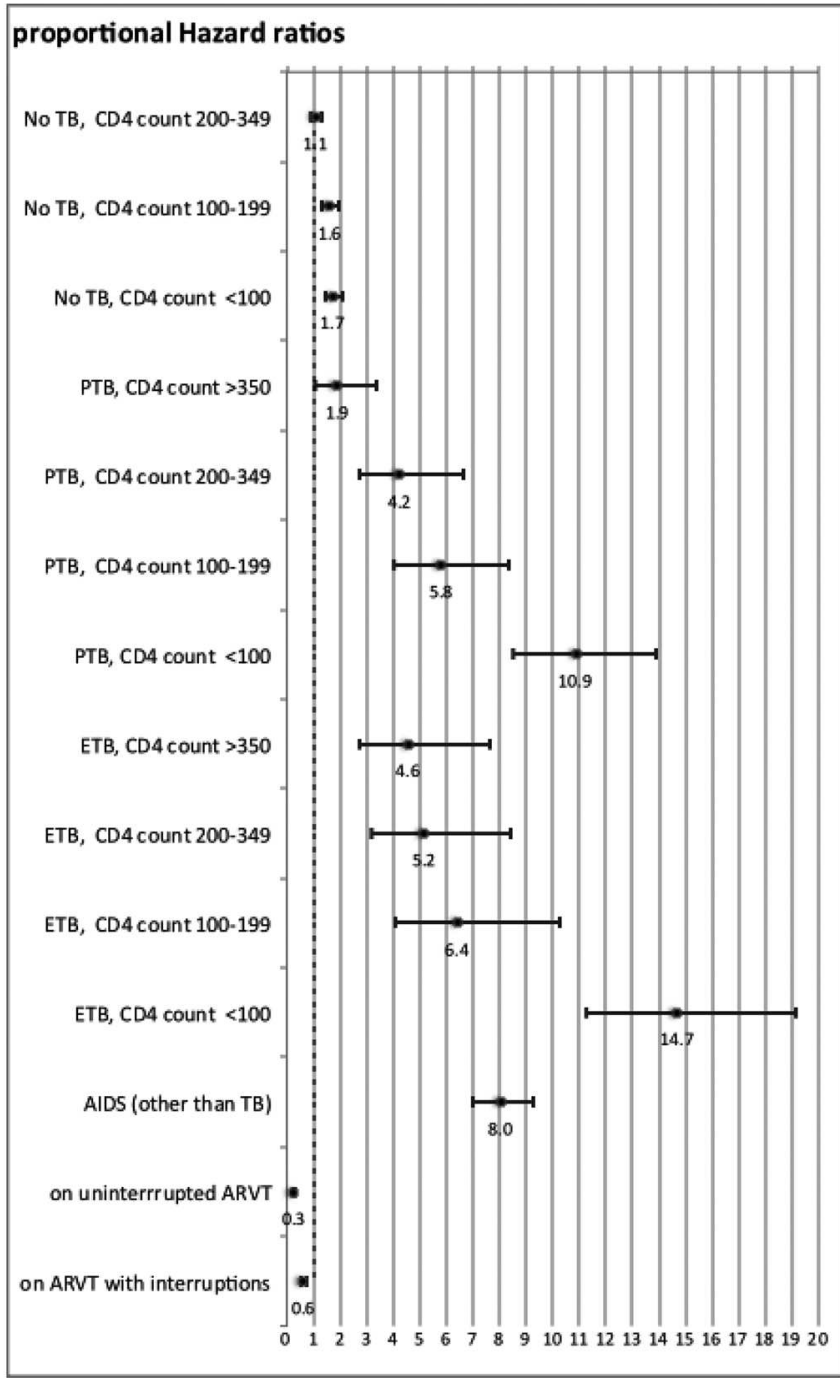

Abstract S58 Figure 1 Multivariable Cox regression model for all cause mortality in persons living with HIV diagnosed in the UK, 2000-2009. Cox regression model for all-cause mortality. There was significant interaction of TB co-infection, CD4 count at diagnosis and site of TB disease-the strata are therefore displayed separately. Dots denote point estimates for hazard ratios for all cause mortality; bars denote $95 \%$ confidence intervals. The model was also adjusted for age, sex, region of birth, and probable route of exposure (not displayed here, as effect sizes are moderate and to keep fewer categories). Abbreviations: PTB-pulmonary TB, ETBextrapulmonary TB, ARVT-Antiretroviral Therapy. The baseline patient is characterised, as a 25-34 year-old UK-born MSM who has never been on ARV, is not TB co-infected and has a CD4 count of 350 or above.
Health England, London, UK; ${ }^{2}$ Erasmus University, Rotterdam, Netherlands; ${ }^{3}$ Chelsea and Westminster Hospital, London, UK

\subsection{6/thoraxinl-2013-204457.65}

Introduction Tuberculosis (TB) rates in the UK are amongst the highest in Western Europe and HIV-TB co-infection (HIV-TB) contributes significantly to the burden of TB in the UK. Globally, HIV-TB is associated with significant morbidity and mortality, in particular where there is delayed diagnosis. Analysis of settingspecific survival can inform local healthcare policy. Here we report the outcome of individuals diagnosed with HIV in the UK, with particular emphasis on TB co-infection.

Methodology We examined deaths among a retrospective national cohort of adults (15 years + ) diagnosed with HIV infection between 2000-2008 linked to the national TB databases and death records from the Office of National Statistics to mid2010. Hazard ratios (HR) estimates using uni- and multi-variable Cox regression modelling were calculated to compare all-cause and AIDS-specific mortality by key demographic and clinical markers.

Results A total of 1,880 (4.3\%) deaths were observed among 44,050 HIV-diagnosed adults during 149,663 person-years of follow-up. 3,188 (7.2\%) adults developed TB and HIV-TB cases accounted for $341(18.1 \%)$ of all deaths of whom $270(79.2 \%)$ were late presenters (CD4 $<200$ at HIV diagnosis). One year mortality after HIV diagnosis was $45 \%$ overall and greater among HIV-TB cases (54\%) and those with low CD4 counts at diagnosis $(69 \%$ for CD4<50). TB co-infection and a low CD4 count at HIV diagnosis significantly increased the hazard of allcause mortality. In the fully adjusted model, the highest HR was among adults with extrapulmonary $\mathrm{TB}$ and pulmonary $\mathrm{TB}$ cases with CD4 count $<100$ at diagnosis (figure1). The Cox model of AIDS-specific mortality showed similar findings, albeit with increased effect size. Increasing age, female sex, UK country of birth, heterosexual route of exposure to HIV and other AIDS conditions were also associated with poor survival. Antiretroviral therapy (ART) was protective, even if interrupted.

Discussion Despite the availability of effective ART and TB screening and treatment, we observed high death rates among a large national HIV cohort associated with late diagnosis and TB infection. The findings highlight the on-going need for expanded HIV testing and increased TB case finding among HIV diagnosed adults to ensure appropriate and early treatment initiation for both conditions.

\section{S59 DRAMATIC DECLINE IN PLASMA SMALL RNA CONCENTRATION IN HIV-INFECTED AND UNINFECTED INDIVIDUALS RECEIVING ANTI-TUBERCULOSIS THERAPY: A PUTATIVE BIOMARKER OF TREATMENT RESPONSE}

I Honeyborne, C Eckold, SH Gillespie, M Lipman, A Pym, TD McHugh; University College London, London, UK

\subsection{6/thoraxjnl-2013-204457.66}

Non-coding RNA molecules, particularly miRNA, regulate translation of mRNA and have been found to target expression of genes important in immune response, such as IFN- $\gamma$. Differences in blood transcriptome signatures and differential miRNA expression have been reported to discriminate between uninfected, active and latent tuberculosis. We analysed the ability of small RNA molecules (0-150 nucleotides) in blood plasma to act as biomarkers of tuberculosis treatment response. Total blood 